\title{
Erythroid cells and malaria parasites: it's a match!
}

\section{Gaëlle Neveu ${ }^{1}$ and Catherine Lavazec ${ }^{2,3}$}

\author{
${ }^{1}$ Department of Microbiology and Immunology, Weill Cornell Medicine, New York, NY, 10065 USA. \\ ${ }^{2}$ Inserm U1016, CNRS UMR8104, Université de Paris, Institut Cochin, Paris, France \\ ${ }^{3}$ Laboratoire d'excellence GR-Ex, Paris, France
}

Correspondence: Catherine Lavazec: catherine.lavazec@inserm.fr

\section{Purpose of review}

This review outlines recent discoveries on the infection of erythroid cells by Plasmodium parasites, focusing on the molecular interactions governing the tropism of parasites for their host cell and the implications of this tropism for parasite biology and erythroid cell maturation.

\section{Recent findings}

Although most studies about the interactions of Plasmodium parasites and their host cell focused on the deadliest human malaria parasite, Plasmodium falciparum, and the erythrocyte, there is increasing evidence that several Plasmodium species, including $P$. falciparum, also develop within erythroid precursors. These interactions likely modify the remodeling of the host cell by the parasite and affect the maturation of erythroblast and reticulocytes.

\section{Summary}

A better understanding of the remodeling of immature erythroid cells by Plasmodium parasites will have important implications for the development of antimalarial drugs or vaccines. In addition, deciphering how Plasmodium parasites interfere with erythropoiesis will provide new insights on how these parasites contribute to anemia in malaria patients.

\section{Keywords}

Malaria, Plasmodium, erythropoiesis, host-parasite interactions, cell tropism 


\section{Key Points}

- Several Plasmodium species preferentially develop within erythroblasts or reticulocytes

- Host cell tropism is dependent on receptors expressed at the surface of erythroid cells and on different intracellular conditions

- Parasite-induced remodeling of the infected host cell is likely different in erythroid precursors than in their mature counterparts

- The development of erythroblasts and reticulocytes is affected by parasite infection

\section{Introduction}

Among all pathogens infecting erythroid cells, the malaria-causing parasite Plasmodium has the highest incidence. This ancestral unicellular eukaryote evolved with the animal kingdom and now infects a wide range of vertebrates and insects. Five species can infect humans: $P$. falciparum, $P$. vivax, P. malariae, P. knowlesi and P. ovale. Among other species infecting animals, rodent malaria parasites, such as $P$. berghei or $P$. yoelii, are used as models for human malaria. In 2018, more than 200 million people contracted malaria and the disease caused approximately 405,000 deaths, mainly in subSaharan Africa [1]. Plasmodium is transmitted to vertebrates by a mosquito, which injects mobile and infective forms of the parasite into the bloodstream from where they go directly to the liver and multiply asymptomatically. After several days, parasites are released into the bloodstream as merozoites and invade erythrocytes where they develop for 42 to 48 hours. Once fully mature, the parasite triggers the rupture of the erythrocyte to release new merozoites, which can again invade red blood cells in the circulation. At each replication cycle, a small portion of the parasites differentiates into male and female sexual forms called gametocytes, the only parasitic form that can be transmitted to mosquitoes. Only immature asexual parasites and mature gametocytes are present in the bloodstream, whereas the other intra-erythrocytic parasitic forms are sequestered in deep tissues [27]. Throughout the intra-erythrocytic cycle, the parasite interacts with and profoundly modifies its red 
blood cell host. The first interactions take place during the invasion process where the parasite binds to proteins on the surface of the erythrocyte, allowing it to enter the cell. Once inside the red blood cell, the parasite, in its parasitic vacuole, makes itself at home: it feeds on the hemoglobin present in the erythrocyte cytoplasm and sets up an export system of its own proteins which will influence the permeability, adhesiveness and deformability of its host cell in order to develop and escape both the immune system and the splenic filtration.

Most studies about the interactions of Plasmodium parasites and their host cells have focused on the development of $P$. falciparum in the mature red blood cell, mainly because it is the most lethal malaria parasite for humans and because it can be cultured with relative ease in the laboratory. However, there is increasing evidence that $P$. falciparum parasites, as well as other Plasmodium species, also interact with more immature cells, the erythroid precursors. Parasites, especially immature gametocytes, have been detected in bone marrow for several decades $[8,9]$ and these observations were recently confirmed by histological and molecular techniques $[2,4,6,10,11]$. More precisely, parasites have been found in proximity to the erythroid niche [6], suggesting that they could interact with erythroblasts and reticulocytes. We discuss here the tropisms of different Plasmodium species and stages for erythroid cells during the erythropoiesis process, as well as the implications of these preferences for both the parasite and its host cell.

\section{Tropism for the red blood cell host}

Plasmodium has been known for decades to multiply in mature red blood cells in the bloodstream, especially since the establishment of an in vitro culture system for $P$. falciparum in human erythrocytes [12]. However, mature erythrocytes are not the only cell type in which the parasite can go through its asexual and sexual replication cycle. Reticulocytes, the enucleated precursors of erythrocytes, appear as a preferential host cell for several Plasmodium species, including P. falciparum [13]. Other species, such as $P$. vivax and $P$. ovale, have a strict tropism for reticulocytes $[14,15]$. $P$. vivax can be only cultivated in these precursor cells [16], due to the recognition of particular receptors present at the 
surface of reticulocytes and not of mature erythrocytes $[17,18]$. In particular, $P$. vivax more easily invades immature reticulocytes still having a strong transferrin receptor 1 (CD71) expression [19]. Rodent parasites, such as $P$. berghei and $P$. yoelii, are also able to develop in murine reticulocytes $[3$, $20,21]$. Although the parasite preference for reticulocytes is not yet fully understood, it has been suggested that the parasite development in reticulocytes increases gametocyte formation [22], which may be related to the presence of sexual parasites in the bone marrow parenchyma. Interestingly, $P$. falciparum gametocytes have been observed within erythroblasts in bone marrow smears [11], suggesting that nucleated erythroid precursors may also be host cells for $P$. falciparum. This observation has been confirmed in vitro, where it is possible to infect erythroid cells from the polychromatic stage and observe the entire parasite asexual development $[2,23]$ as well as the whole gametocytogenesis [11]. P. vivax has also been detected in erythroblasts on bone marrow smears from malaria patients [24], suggesting that this tropism exists in several Plasmodium species. Finally, some murine parasites can also develop in nucleated erythroid precursors: $P$. yoelii has been reported to be able to infect erythroblasts, resulting in the activation of $\mathrm{CD} 8+$ lymphocytes [25]. In contrast, P. berghei does not appear to be able to parasitize these cells [26].

\section{Molecular and cellular interactions underlying the tropism of Plasmodium parasites for erythroid} cells

The ability of Plasmodium parasites to identify their host cell first relies on molecular interactions during the initial steps of invasion between parasite ligands expressed at the apical pole of merozoites and receptors on the red blood cell surface. Two families of parasite adhesins are considered as the primary determinants of host cell tropism: the Erythrocyte-Binding-Like (EBL) protein family and the Reticulocyte-Binding-Like (RBL) protein family [27]. All Plasmodium species encode multiple EBL and RBL proteins, thus providing a high degree of functional redundancy during invasion [27]. Although they play a prominent role in Plasmodium biology, the receptors of most EBL and RBL proteins are still unknown. All identified receptors for $P$. falciparum ligands, such as basigin or glycophorins, are present 
at the surface of erythroid cells from the early stages of erythropoiesis [28], thus enabling $P$. falciparum merozoites to invade both erythroblasts, reticulocytes and mature erythrocytes. For $P$. vivax, the first described ligand-receptor couple was the P. vivax-Duffy-Binding-Protein (PvDBP, belonging to the EBL family) and the Duffy antigen $[18,29]$. However, the presence of Duffy on the surface of mature erythrocytes implies that the strict preference of $P$. vivax for invasion into reticulocytes relies on other receptor(s)[30]. Further genomic studies suggest that the tropism of $P$. vivax for reticulocytes is rather mediated by the RBL family [31]. Subsequent biochemical evidence demonstrates a direct interaction between PVRBP2b and CD71, therefore identifying the second validated host receptor for $P$. vivax [17]. As CD71 is lost during reticulocyte maturation, it is thought to be the main determinant of $P$. vivax tropism for young reticulocytes [19]. In addition to the specific interactions between the parasite and the host cell during invasion, the unique environment within the host cell is also a critical feature for parasite propagation. For instance, the preference of several Plasmodium species for reticulocytes may reflect their requirement of high intracellular levels of glucose-6-phosphate dehydrogenase (G6PD) [32]. This enzyme, which provides a protection from oxidative stress for parasites, is expressed at higher levels in reticulocytes than in mature erythrocytes [33]. In support of this hypothesis, it has been shown that G6PD deficiency confers a protective effect against $P$. vivax [34]. Several Plasmodium species may also prefer immature erythroid cells because the erythroid niche in the bone marrow parenchyma provides an environment where the concentration of oxygen and/or crucial metabolites is different from that in other tissues. Finally, we propose that the development of $P$. falciparum gametocytes within erythroblasts naturally adhering to a macrophage in the erythroblastic islands may reflect a strategy developed by the parasite to sequester in bone marrow [11].

\section{Different home, different life?}

While $P$. falciparum asexual and sexual stages can both develop in erythroid cells from the polychromatic erythroblast stage to the mature erythrocyte, the maturity of the host cell likely affects parasite biology. The hemoglobin content of the erythroid cell, which is a crucial determinant for 
parasite development [35], progressively increases upon erythropoiesis [28]. Thus, the very low hemoglobin content in immature erythroid stages may explain why parasites are not able to develop before the polychromatic stages $[11,23]$. Low hemoglobin concentration likely also influences the susceptibility of Plasmodium parasites to different antimalarials whose mechanism of action depends on the degradation of hemoglobin, as is the case with artemisinin $[36,37]$. The export of proteins by the parasite to its host cell may also differ depending on the maturity of the erythroid cell. In infected mature erythrocytes, it is well known that Plasmodium parasites export hundreds of proteins to their host cell, thereby modifying its structural and mechanical properties. This remodeling drastically changes the cytoadherence, deformability and permeability properties of the erythroid cell $[38,39]$. Because erythrocytes are devoid of internal membranes, this export is ensured by a parasite-derived machinery composed of a vacuolar secretion system, called Plasmodium translocon of exported proteins (PTEX), which transports parasite proteins across the parasitophorous vacuolar membrane, and of membranous structures in the host cell cytosol, called Maurer's clefts $[38,40]$. It remains unknown whether the parasites also export their proteins to the erythroblast cytosol and membrane, and if so, whether they establish this trafficking network within erythroblasts as they do within erythrocytes. In an alternative scenario, one could speculate that they rather hijack the erythroblast endoplasmic reticulum and Golgi apparatus to export their proteins to the erythroblast membrane. Such a different export mechanism may change the localization of parasite proteins in the host cell. Furthermore, the continuous change in membrane protein content and membrane-skeleton organization during erythropoiesis, through endocytosis and membrane loss $[28,41,42]$ may also drastically impact their localization at the erythroblast membrane. Consequently, it is unknown whether parasite proteins exhibit the same localization and surface exposure in infected erythroblasts and in infected erythrocytes. This may result in a different parasite-induced remodeling of the host cell that plays a key role for parasite survival, virulence and recognition by the immune system. In asexual stages, for instance, antigenically variable proteins are exposed on structures protruding from the surface of the infected erythrocyte, called knobs, where they bind to host endothelial receptors. These 
interactions enable the infected erythrocyte to efficiently adhere to the endothelial cells of the microvasculature and thus sequester in deep tissues to evade splenic clearance. Such membrane modifications may not be necessary in erythroblasts that do not circulate and adhere to a nursing macrophage within erythroblastic islands localized in the extra-vascular space of the bone marrow. For the same reason, the deformability of infected erythroid precursors may not be affected like that of mature erythrocytes, since the parasite-induced increase in erythrocyte rigidity has been proposed to promote sequestration of mature asexual and immature sexual stages [43, 44]. Although these changes in infected erythrocyte deformability probably also play other as yet undefined roles, the already significant rigidity of reticulocytes and erythroblasts $[42,45]$ could render unnecessary the mechanisms established by the parasite to regulate the deformability of its host cell $[46,47]$. In addition, it remains unclear whether the parasite-induced increase in erythrocyte membrane permeability, known as the new permeability pathways $[48,49]$, also occurs in the erythroblast, which contains more transport/channel proteins than their mature counterparts [28]. Importantly, new permeability pathways have been shown to contribute to the uptake of several antimalarial drugs by asexual and sexual parasites $[48,50,51]$. Therefore, Plasmodium susceptibility to known antimalarials must be re-evaluated considering parasite development in different cells than those already described. Likewise, a different localization or surface exposure of several parasite proteins in immature erythroid cells could jeopardize the development of some vaccine candidates.

\section{Parasite impact on host cell development}

The consequences of parasitic infection differ depending on the maturity of the erythroid cell. Upon infection with $P$. vivax, the biomechanically rigid immature CD71-positive reticulocyte converts into a highly deformable CD71-negative mature red cell devoid of host reticular matter within 3 to 6 hours, whereas this process normally lasts 24 hours for uninfected reticulocytes [19]. It has been proposed that this parasite-induced accelerated aging of immature reticulocytes and the resulting increased deformability may be an adaptation that would enable the parasitized cell to egress the bone marrow 
and to escape splenic clearance $[19,52,53]$. Conversely, we recently observed that $P$. falciparum gametocytes delay the erythroblasts development [11]. Although erythroblast infection is not possible before the polychromatic stage, gametocytes slow down the differentiation of erythroblasts into reticulocytes in order to complete their 10-day development in nucleated cells. P. falciparum parasites may have developed this strategy to allow gametocyte maturation to coincide with the release of their host cell from bone marrow. After the release of mature gametocyte-infected reticulocytes in peripheral blood, it remains to be determined whether $P$. falciparum infection hastens the maturation of reticulocytes and the loss of CD71 as observed in P. vivax-infected cells. Such rapid remodeling may be a general feature of Plasmodium spp. infection that enables parasites to circulate across the spleen. Impaired erythropoiesis observed in infected erythroblasts is triggered both by the direct effect of gametocytes on their host cell and by the release of parasite-derived extracellular vesicles, which in both cases increase the oxidative stress in erythroblasts. As oxidative stress can lead to inhibition of erythroid maturation and dyserythropoiesis [54], such an increase in oxidation may be responsible for the reported delay in erythropoiesis. Erythroblast infection and extracellular vesicles secreted in the bone marrow parenchyma may therefore be involved in erythroid disorders in malaria patients. This hypothesis is supported by the observation of ineffective erythropoiesis in patients infected with $P$. falciparum and P. vivax [55-59]. Although less data is available on rodent parasites, some studies also suggest that $P$. berghei affects erythropoiesis in infected mice $[26,60]$. Deciphering how Plasmodium parasites interfere with erythropoiesis will be a challenge for future studies in this field.

\section{Conclusion}

Investigating Plasmodium development in erythroid precursors is complicated by the scarcity and difficulty of culturing primary erythroid cells. The use of immortalized erythroid cell lines could be a good alternative to further study the asexual and sexual development of the parasite in these cells. Several teams have succeeded in immortalizing erythroid precursors to make them differentiate up to the reticulocyte stage in vitro, producing different cell models such as JK1, bel-A and EJ cells [61-63]. 
These models have so far been primarily used for the study of the various erythroid receptors involved in parasite invasion, which may be invalidated by CRISPR-Cas9 in these nucleated cells. Although these models do not exactly mimic the process of erythropoiesis and do not produce large amounts of reticulocytes, they will surely be a powerful tool for studying how parasites remodel their host cell. Expected results will have crucial implications for the development of antimalarial drugs or vaccines, which must be reassessed in light of the parasite development in erythroid precursors. On the other hand, unravelling how Plasmodium parasites interfere with erythroid development will still require the use of primary erythroid cells. However, this important research will provide new insights into the processes of ineffective erythropoiesis in malaria patients, and may lead to new therapeutic strategies to treat malarial anemia.

\section{Acknowledgements}

We thank all contributors to this research topic and apologize to authors whose work could not be cited due to space limitations. We thank Frédérique Verdier for critical reading and Kelly Harding for English editing of the manuscript.

\section{Financial support and sponsorship}

The authors acknowledge the financial support from the Cnrs, Inserm, the Fondation pour la Recherche Médicale (“Equipe FRM” grant DEQ20170336722) and the Laboratory of Excellence GR-Ex, reference ANR-11-LABX0051, funded by the program 'Investissements d'avenir' of the French National Research Agency, reference ANR11-IDEX-0005-02.

\section{Conflicts of interest}

The authors declare no competing interests. 


\section{References}

Papers of particular interest, published within the period of review, have been highlighted as:

- of special interest

- of outstanding interest

1. WHO (2019). World Malaria Report 2019.

2. Aguilar, R., Magallon-Tejada, A., Achtman, A.H., Moraleda, C., Joice, R., Cistero, P., Li Wai Suen, C.S., Nhabomba, A., Macete, E., Mueller, I., et al. (2014). Molecular evidence for the localization of Plasmodium falciparum immature gametocytes in bone marrow. Blood 123, 959-966.

3. De Niz, M., Meibalan, E., Mejia, P., Ma, S., Brancucci, N.M.B., Agop-Nersesian, C., Mandt, R., Ngotho, P., Hughes, K.R., Waters, A.P., et al. (2018). Plasmodium gametocytes display homing and vascular transmigration in the host bone marrow. Sci Adv 4, eaat3775.

- Intra-vital imaging on Plasmodium berhei-infected mice to observe the immature gametocyte homing in the bone marrow parenchyma.

4. Farfour, E., Charlotte, F., Settegrana, C., Miyara, M., and Buffet, P. (2012). The extravascular compartment of the bone marrow: a niche for Plasmodium falciparum gametocyte maturation? Malar J 11, 285.

5. Franke-Fayard, B., Janse, C.J., Cunha-Rodrigues, M., Ramesar, J., Buscher, P., Que, I., Lowik, C., Voshol, P.J., den Boer, M.A., van Duinen, S.G., et al. (2005). Murine malaria parasite sequestration: CD36 is the major receptor, but cerebral pathology is unlinked to sequestration. Proc Natl Acad Sci U S A 102, 11468-11473.

6. Joice, R., Nilsson, S.K., Montgomery, J., Dankwa, S., Egan, E., Morahan, B., Seydel, K.B., Bertuccini, L., Alano, P., Williamson, K.C., et al. (2014). Plasmodium falciparum transmission stages accumulate in the human bone marrow. Sci Transl Med 6, 244 re245.

- This study validates the presence of Plasmodium falciparum gametocytes in the bone marrow parenchyma and showed that the parasite can infect erythroblasts in vitro.

7. Obaldia, N., 3rd, Meibalan, E., Sa, J.M., Ma, S., Clark, M.A., Mejia, P., Moraes Barros, R.R., Otero, W., Ferreira, M.U., Mitchell, J.R., et al. (2018). Bone Marrow Is a Major Parasite Reservoir in Plasmodium vivax Infection. mBio 9.

- This study validates the presence of Plasmodium vivax in the bone marrow parenchyma.

8. Smalley, M.E., Abdalla, S., and Brown, J. (1981). The distribution of Plasmodium falciparum in the peripheral blood and bone marrow of Gambian children. Trans R Soc Trop Med Hyg 75, 103-105.

9. Wickramasinghe, S.N., Phillips, R.E., Looareesuwan, S., Warrell, D.A., and Hughes, M. (1987). The bone marrow in human cerebral malaria: parasite sequestration within sinusoids. $\mathrm{Br} \mathrm{J}$ Haematol 66, 295-306.

10. Baro, B., Deroost, K., Raiol, T., Brito, M., Almeida, A.C., de Menezes-Neto, A., Figueiredo, E.F., Alencar, A., Leitao, R., Val, F., et al. (2017). Plasmodium vivax gametocytes in the bone marrow of an acute malaria patient and changes in the erythroid miRNA profile. PLoS NegI Trop Dis 11, e0005365. 
11. Neveu, G., Richard, C., Dupuy, F., Behera, P., Volpe, F., Subramani, P.A., Marcel-Zerrougui, B., Vallin, P., Andrieu, M., Minz, A.M., et al. (2020). Plasmodium falciparum sexual parasites develop in human erythroblasts and affect erythropoiesis. Blood 136, 1381-1393.

- Human erythroblasts were identified as hosts for the complete gametocyte development of Plasmodium falciparum, resulting in a delay in erythropoiesis.

12. Trager, W., and Jensen, J.B. (1976). Human malaria parasites in continuous culture. Science $193,673-675$.

13. Pasvol, G., Weatherall, D.J., and Wilson, R.J. (1980). The increased susceptibility of young red cells to invasion by the malarial parasite Plasmodium falciparum. Br J Haematol 45, 285-295.

14. Kitchen, S. (1938). The Infection of Reticulocytes by Plasmodium Vivax. The American Journal of Tropical Medicine and Hygiene s1-18, 347-359.

15. Russell, B.M., and Cooke, B.M. (2017). The Rheopathobiology of Plasmodium vivax and Other Important Primate Malaria Parasites. Trends Parasitol 33, 321-334.

16. Golenda, C.F., Li, J., and Rosenberg, R. (1997). Continuous in vitro propagation of the malaria parasite Plasmodium vivax. Proc Natl Acad Sci U S A 94, 6786-6791.

17. Gruszczyk, J., Kanjee, U., Chan, L.J., Menant, S., Malleret, B., Lim, N.T.Y., Schmidt, C.Q., Mok, Y.F., Lin, K.M., Pearson, R.D., et al. (2018). Transferrin receptor 1 is a reticulocyte-specific receptor for Plasmodium vivax. Science 359, 48-55.

- This study identifies CD71 as a new receptor for Plasmodium vivax invasion in reticulocytes.

18. Miller, L.H., Mason, S.J., Clyde, D.F., and McGinniss, M.H. (1976). The resistance factor to Plasmodium vivax in blacks. The Duffy-blood-group genotype, FyFy. N Engl J Med 295, 302304.

19. Malleret, B., Li, A., Zhang, R., Tan, K.S., Suwanarusk, R., Claser, C., Cho, J.S., Koh, E.G., Chu, C.S., Pukrittayakamee, S., et al. (2015). Plasmodium vivax: restricted tropism and rapid remodeling of CD71-positive reticulocytes. Blood 125, 1314-1324.

- This article reports the tropism of Plasmodium vivax for CD71-positive young reticulocytes and the resulting accelerated maturation of these cells upon infection.

20. Cromer, D., Evans, K.J., Schofield, L., and Davenport, M.P. (2006). Preferential invasion of reticulocytes during late-stage Plasmodium berghei infection accounts for reduced circulating reticulocyte levels. Int J Parasitol 36, 1389-1397.

21. Vigario, A.M., Belnoue, E., Cumano, A., Marussig, M., Miltgen, F., Landau, I., Mazier, D., Gresser, I., and Renia, L. (2001). Inhibition of Plasmodium yoelii blood-stage malaria by interferon alpha through the inhibition of the production of its target cell, the reticulocyte. Blood 97, 3966-3971.

22. Trager, W., Gill, G.S., Lawrence, C., and Nagel, R.L. (1999). Plasmodium falciparum: enhanced gametocyte formation in vitro in reticulocyte-rich blood. Exp Parasitol 91, 115-118.

23. Tamez, P.A., Liu, H., Fernandez-Pol, S., Haldar, K., and Wickrema, A. (2009). Stage-specific susceptibility of human erythroblasts to Plasmodium falciparum malaria infection. Blood 114, 3652-3655.

- This study shows that Plasmodium falciparum can infect nucleated erythroblasts in vitro, preferentially from the ortochromatic stage of development.

24. Ru, Y.X., Mao, B.Y., Zhang, F.K., Pang, T.X., Zhao, S.X., Liu, J.H., and Wickramasinghe, S.N. (2009). Invasion of erythroblasts by Pasmodium vivax: A new mechanism contributing to malarial anemia. Ultrastruct Pathol 33, 236-242. 
-. Two case reports demonstrating the presence of Plasmodium vivax parasites within erythroblasts in the bone marrow of malaria-infected patients.

25. Imai, T., Ishida, H., Suzue, K., Hirai, M., Taniguchi, T., Okada, H., Suzuki, T., Shimokawa, C., and Hisaeda, H. (2013). CD8(+) T cell activation by murine erythroblasts infected with malaria parasites. Sci Rep 3, 1572.

26. Lakkavaram, A., Lundie, R.J., Do, H., Ward, A.C., and de Koning-Ward, T.F. (2020). Acute Plasmodium berghei Mouse Infection Elicits Perturbed Erythropoiesis With Features That Overlap With Anemia of Chronic Disease. Front Microbiol 11, 702.

- This article describes the impact of Plasmodium berghei infection on murine erythroblasts maturation.

27. Iyer, J., Gruner, A.C., Renia, L., Snounou, G., and Preiser, P.R. (2007). Invasion of host cells by malaria parasites: a tale of two protein families. Mol Microbiol 65, 231-249.

28. Gautier, E.F., Ducamp, S., Leduc, M., Salnot, V., Guillonneau, F., Dussiot, M., Hale, J., Giarratana, M.C., Raimbault, A., Douay, L., et al. (2016). Comprehensive Proteomic Analysis of Human Erythropoiesis. Cell Rep 16, 1470-1484.

29. Barnwell, J.W., Nichols, M.E., and Rubinstein, P. (1989). In vitro evaluation of the role of the Duffy blood group in erythrocyte invasion by Plasmodium vivax. J Exp Med 169, 1795-1802.

30. Malleret, B., Xu, F., Mohandas, N., Suwanarusk, R., Chu, C., Leite, J.A., Low, K., Turner, C., Sriprawat, K., Zhang, R., et al. (2013). Significant biochemical, biophysical and metabolic diversity in circulating human cord blood reticulocytes. PLoS One 8, e76062.

31. Carlton, J.M., Adams, J.H., Silva, J.C., Bidwell, S.L., Lorenzi, H., Caler, E., Crabtree, J., Angiuoli, S.V., Merino, E.F., Amedeo, P., et al. (2008). Comparative genomics of the neglected human malaria parasite Plasmodium vivax. Nature 455, 757-763.

32. Gunalan, K., Rowley, E.H., and Miller, L.H. (2020). A Way Forward for Culturing Plasmodium vivax. Trends Parasitol 36, 512-519.

33. Wilson, M.C., Trakarnsanga, K., Heesom, K.J., Cogan, N., Green, C., Toye, A.M., Parsons, S.F., Anstee, D.J., and Frayne, J. (2016). Comparison of the Proteome of Adult and Cord Erythroid Cells, and Changes in the Proteome Following Reticulocyte Maturation. Mol Cell Proteomics 15, 1938-1946.

34. Louicharoen, C., Patin, E., Paul, R., Nuchprayoon, I., Witoonpanich, B., Peerapittayamongkol, C., Casademont, I., Sura, T., Laird, N.M., Singhasivanon, P., et al. (2009). Positively selected G6PD-Mahidol mutation reduces Plasmodium vivax density in Southeast Asians. Science 326, 1546-1549.

35. Liu, J., Istvan, E.S., Gluzman, I.Y., Gross, J., and Goldberg, D.E. (2006). Plasmodium falciparum ensures its amino acid supply with multiple acquisition pathways and redundant proteolytic enzyme systems. Proc Natl Acad Sci U S A 103, 8840-8845.

36. Birnbaum, J., Scharf, S., Schmidt, S., Jonscher, E., Hoeijmakers, W.A.M., Flemming, S., Toenhake, C.G., Schmitt, M., Sabitzki, R., Bergmann, B., et al. (2020). A Kelch13-defined endocytosis pathway mediates artemisinin resistance in malaria parasites. Science 367, 51-59.

37. Wicht, K.J., Mok, S., and Fidock, D.A. (2020). Molecular Mechanisms of Drug Resistance in Plasmodium falciparum Malaria. Annu Rev Microbiol 74, 431-454.

38. de Koning-Ward, T.F., Gilson, P.R., Boddey, J.A., Rug, M., Smith, B.J., Papenfuss, A.T., Sanders, P.R., Lundie, R.J., Maier, A.G., Cowman, A.F., et al. (2009). A newly discovered protein export machine in malaria parasites. Nature 459, 945-949. 
39. Neveu, G., and Lavazec, C. (2019). Erythrocyte Membrane Makeover by Plasmodium falciparum Gametocytes. Frontiers in Microbiology 10.

40. Haldar, K., Mohandas, N., Samuel, B.U., Harrison, T., Hiller, N.L., Akompong, T., and Cheresh, P. (2002). Protein and lipid trafficking induced in erythrocytes infected by malaria parasites. Cell Microbiol 4, 383-395.

41. Minetti, G., Achilli, C., Perotti, C., and Ciana, A. (2018). Continuous Change in Membrane and Membrane-Skeleton Organization During Development From Proerythroblast to Senescent Red Blood Cell. Front Physiol 9, 286.

42. Chu, T.T.T., Sinha, A., Malleret, B., Suwanarusk, R., Park, J.E., Naidu, R., Das, R., Dutta, B., Ong, S.T., Verma, N.K., et al. (2018). Quantitative mass spectrometry of human reticulocytes reveal proteome-wide modifications during maturation. $\mathrm{Br} J$ Haematol 180, 118-133.

43. Sanyal, S., Egee, S., Bouyer, G., Perrot, S., Safeukui, I., Bischoff, E., Buffet, P., Deitsch, K.W., Mercereau-Puijalon, O., David, P.H., et al. (2012). Plasmodium falciparum STEVOR proteins impact erythrocyte mechanical properties. Blood 119, e1-8.

44. Silvestrini, F., Tiburcio, M., Bertuccini, L., and Alano, P. (2012). Differential adhesive properties of sequestered asexual and sexual stages of Plasmodium falciparum on human endothelial cells are tissue independent. PLoS One 7, e31567.

45. Leblond, P.F., LaCelle, P.L., and Weed, R.I. (1971). Cellular deformability: a possible determinant of the normal release of maturing erythrocytes from the bone marrow. Blood 37, 40-46.

46. Naissant, B., Dupuy, F., Duffier, Y., Lorthiois, A., Duez, J., Scholz, J., Buffet, P., Merckx, A., Bachmann, A., and Lavazec, C. (2016). Plasmodium falciparum STEVOR phosphorylation regulates host erythrocyte deformability enabling malaria parasite transmission. Blood 127, e42-53.

47. Ramdani, G., Naissant, B., Thompson, E., Breil, F., Lorthiois, A., Dupuy, F., Cummings, R., Duffier, Y., Corbett, Y., Mercereau-Puijalon, O., et al. (2015). cAMP-Signalling Regulates Gametocyte-Infected Erythrocyte Deformability Required for Malaria Parasite Transmission. PLoS Pathog 11, e1004815.

48. Bouyer, G., Barbieri, D., Dupuy, F., Marteau, A., Sissoko, A., N'Dri, M.E., Neveu, G., Bedault, L., Khodabux, N., Roman, D., et al. (2020). Plasmodium falciparum sexual parasites regulate infected erythrocyte permeability. Commun Biol 3, 726.

49. Ginsburg, H., Krugliak, M., Eidelman, O., and Cabantchik, Z.I. (1983). New permeability pathways induced in membranes of Plasmodium falciparum infected erythrocytes. Mol Biochem Parasitol 8, 177-190.

50. Baumeister, S., Wiesner, J., Reichenberg, A., Hintz, M., Bietz, S., Harb, O.S., Roos, D.S., Kordes, M., Friesen, J., Matuschewski, K., et al. (2011). Fosmidomycin uptake into Plasmodium and Babesia-infected erythrocytes is facilitated by parasite-induced new permeability pathways. PLoS One 6, e19334.

51. Dana, S., Prusty, D., Dhayal, D., Gupta, M.K., Dar, A., Sen, S., Mukhopadhyay, P., Adak, T., and Dhar, S.K. (2014). Potent antimalarial activity of acriflavine in vitro and in vivo. ACS Chem Biol 9, 2366-2373.

52. Handayani, S., Chiu, D.T., Tjitra, E., Kuo, J.S., Lampah, D., Kenangalem, E., Renia, L., Snounou, G., Price, R.N., Anstey, N.M., et al. (2009). High deformability of Plasmodium vivax-infected red blood cells under microfluidic conditions. J Infect Dis 199, 445-450. 
53. Suwanarusk, R., Cooke, B.M., Dondorp, A.M., Silamut, K., Sattabongkot, J., White, N.J., and Udomsangpetch, R. (2004). The deformability of red blood cells parasitized by Plasmodium falciparum and P. vivax. J Infect Dis 189, 190-194.

54. Zhao, B., Mei, Y., Yang, J., and Ji, P. (2016). Erythropoietin-regulated oxidative stress negatively affects enucleation during terminal erythropoiesis. Exp Hematol 44, 975-981.

55. Abdalla, S.H., Wickramasinghe, S.N., and Weatherall, D.J. (1984). The deoxyuridine suppression test in severe anaemia following Plasmodium falciparum malaria. Trans R Soc Trop Med Hyg 78, 60-63.

56. Brito, M.A.M., Baro, B., Raiol, T.C., Ayllon-Hermida, A., Safe, I.P., Deroost, K., Figueiredo, E.F.G., Costa, A.G., Armengol, M.D.P., Sumoy, L., et al. (2020). Morphological and Transcriptional Changes in Human Bone Marrow During Natural Plasmodium vivax Malaria Infections. J Infect Dis.

57. Dormer, P., Dietrich, M., Kern, P., and Horstmann, R.D. (1983). Ineffective erythropoiesis in acute human $P$. falciparum malaria. Blut 46, 279-288.

58. Wickramasinghe, S.N., Abdalla, S., and Weatherall, D.J. (1982). Cell cycle distribution of erythroblasts in P. falciparum malaria. Scand J Haematol 29, 83-88.

59. Wickramasinghe, S.N., Looareesuwan, S., Nagachinta, B., and White, N.J. (1989). Dyserythropoiesis and ineffective erythropoiesis in Plasmodium vivax malaria. Br J Haematol 72, 91-99.

60. Maggio-Price, L., Brookoff, D., and Weiss, L. (1985). Changes in hematopoietic stem cells in bone marrow of mice with Plasmodium berghei malaria. Blood 66, 1080-1085.

61. Kanjee, U., Gruring, C., Chaand, M., Lin, K.M., Egan, E., Manzo, J., Jones, P.L., Yu, T., Barker, R., Jr., Weekes, M.P., et al. (2017). CRISPR/Cas9 knockouts reveal genetic interaction between strain-transcendent erythrocyte determinants of Plasmodium falciparum invasion. Proc Natl Acad Sci U S A 114, E9356-E9365.

62. Satchwell, T.J., Wright, K.E., Haydn-Smith, K.L., Sanchez-Roman Teran, F., Moura, P.L., Hawksworth, J., Frayne, J., Toye, A.M., and Baum, J. (2019). Genetic manipulation of cell line derived reticulocytes enables dissection of host malaria invasion requirements. Nat Commun $10,3806$.

63. Scully, E.J., Shabani, E., Rangel, G.W., Gruring, C., Kanjee, U., Clark, M.A., Chaand, M., Kurita, R., Nakamura, Y., Ferreira, M.U., et al. (2019). Generation of an immortalized erythroid progenitor cell line from peripheral blood: A model system for the functional analysis of Plasmodium spp. invasion. Am J Hematol. 